\title{
A novel approach for detecting HMDSO poisoning of metal oxide gas sensors and improving their stability by temperature cycled operation
}

\author{
M. Schüler, T. Sauerwald, and A. Schütze \\ Laboratory for Measurement Technology, Saarland University, Saarbrücken, Germany \\ Correspondence to: M. Schüler (m.schueler@lmt.uni-saarland.de)
}

Received: 10 July 2015 - Revised: 28 September 2015 - Accepted: 3 October 2015 - Published: 19 October 2015

\begin{abstract}
In this paper we study the effect of hexamethyldisiloxane (HMDSO) vapor on an $\mathrm{SnO}_{2}$-based gas sensor (GGS 1330, UST Umweltsensortechnik GmbH, Geschwenda, Germany) in a temperature cycled operation (TCO). We show that HMDSO poisoning can be quantified at early stages ( 85 to $340 \mathrm{ppm} \times$ min) with a resolution of $\pm 85 \mathrm{ppm} \times$ min using TCO. This novel approach for sensor self-monitoring provides a simple method for early detection of HMDSO poisoning. It is thereby possible to detect poisoning before the sensor function is strongly impaired. In this paper we show that by using an appropriate normalization of the sensor data, the stability of gas discrimination by linear discriminant analysis (LDA) can be improved, which in turn facilitates a more accurate determination of the poisoning state by a hierarchical LDA discrimination.

For a specific temperature cycle and feature extraction approach, we show that identification of ethanol and carbon monoxide is still possible after poisoning with $900 \mathrm{ppm} \times$ min HMDSO, i.e. a HMDSO poisoning dose more than twice as high as required by DIN EN 50194-1.
\end{abstract}

\section{Introduction}

Metal oxide semiconductor (MOS) gas sensors are available at relatively low cost and can detect a broad range of reducing and oxidizing gases with high sensitivity. They take advantage of a resistance change, usually in a granular metal oxide. The resistance of the metal oxide semiconductor is increased by the adsorption of oxygen at the interface between grains. The underlying mechanism is described in a well-known model, which states that the oxygen creates a depletion layer that acts as an energy barrier determining the resistance of the material (Morrison, 1982; Kohl, 1989). Typically, in order to enhance the sensitivity of the sensors catalysts, e.g., platinum or palladium, are added. For commercial sensors only the base material is usually communicated; the composition of the catalyst or other additives is normally not published for obvious reasons.

Chemical sensors like MOS are prone to poisoning by irreversible adsorption as the sensor principle involves a direct chemical interaction between sensor and environment. Especially volatile siloxanes, e.g. hexamethyldisilox- ane (HMDSO), are of concern as they can generate nonvolatile (siliceous) aggregates on reactive surfaces and its catalysts (Ehrhardt et al., 1997). This has been shown to change the sensor properties, such as sensitivity and selectivity, which can be attributed to the changing density of active oxygen adsorption sites at the sensor surface (Williams, 1999).

Organic silicones that emit volatile siloxane have many applications and can be found in almost any environment. Durability against poisoning is an important property of a sensor system. Therefore, several standards, e.g. DIN EN 50194-1, describe test methods for detectors of combustible gases in domestic premises. DIN EN 50194-1 requires such detectors to still operate properly after being exposed to $10 \pm 3 \mathrm{ppm}$ HMDSO over a time period of $40 \mathrm{~min}$, corresponding to a dose of $400 \pm 120 \mathrm{ppm} \times$ min.

The sensing properties derived from the interaction of adsorbed oxygen with reducing or oxidizing gases depend strongly on the temperature (Morrison, 1987). This dependency can be exploited to enhance selectivity, leading to the concept of temperature cycled operation (TCO) (Eicker et 


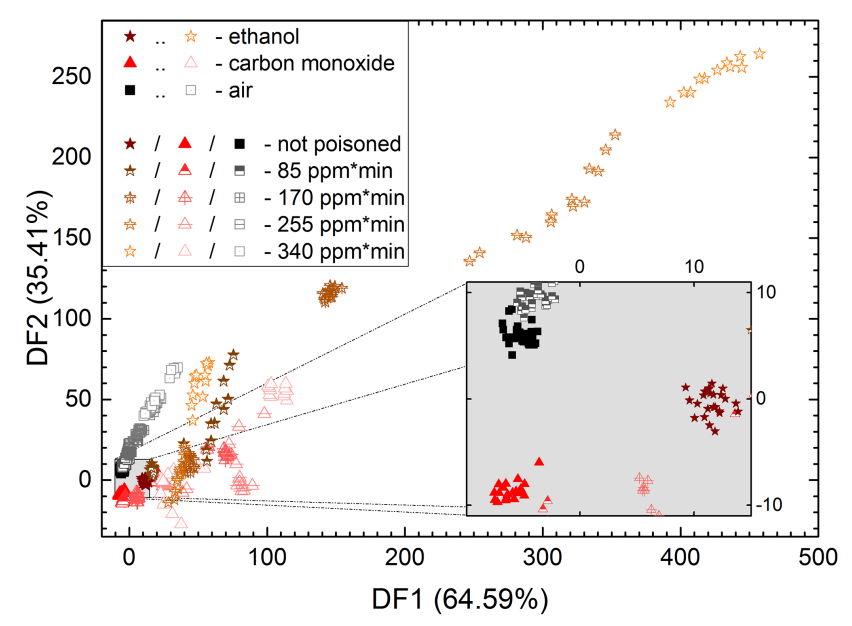

Figure 1. LDA plot showing the discrimination of different gases and data from different poisoning states. The DFs were calculated with data acquired before poisoning (solid symbols), and applied to data from all poisoning states. The 24 features used for this LDA are based on non-normalized conductance values.

al. 1981; Lalauze and Pijolat, 1984; Lee and Ready, 1999; Gramm and Schütze, 2003), which is based on the evaluation of conductance over time during a temperature profile.

In TCO, the sensor temperature is varied in a defined manner, allowing for the study of the sensor's dynamic resistance properties during fast temperature changes as well as at defined temperature levels. It has been shown that TCO can increase selectivity decisively (Gramm and Schütze, 2003; Meier et al., 2007; Leidinger et al., 2014). Furthermore, it can also increase the sensitivity of MOS gas sensors dramatically (Baur et al., 2015) as well as improve the stability of MOX-based sensor systems (Schütze et al., 2004).

In a previous study, we examined the possibility of detecting sensor impairments, such as poisoning by HMDSO, by combining TCO with electrical impedance spectroscopy (EIS) (Schüler et al., 2014). For this combination, a high-speed impedance spectroscope is required. We developed an inexpensive system, which was successfully tested for the detection of HMDSO poisoning (Schüler et al., 2014). However, the hardware required for combined TCOEIS measurements is still quite costly, especially compared to the one required for DC-resistance measurements in TCO mode only.

\section{Detecting HMDSO poisoning using TCO}

We have previously presented a strategy to detect sensor poisoning, and examined how TCO and an appropriate data processing approach can be used to enhance the stability of gas discrimination and facilitate an accurate determination of the poisoning state (Schüler et al., 2015). For the discrimination of different gases and the detection of poisoning, multivariate analysis of the conductance profile was employed.
Gas tests were performed with carbon monoxide (CO) and ethanol at concentrations of a few ppm. Carbon monoxide in the lower ppm range is a typical target gas for MOS gas sensors in safety applications like fire detection (Kohl et al., 2001), while ethanol is a commonly occurring interference gas in many environments. Further experimental details are given in Schüler et al., 2015.

We found that HMDSO poisoning has a strong influence on the absolute conductance, while the shape of the conductance profile remains nearly unchanged and should therefore provide a more stable signal than the absolute conductance value. Thus, the influence of a normalization of the conductance profile to the discrimination of different gases was examined. For normalization, we perform a linear projection of the conductance values of each temperature cycle, assigning the cycle's lowest conductance value to 0 and the highest value to 1 . To enable multivariate analysis of the sensor data, it is then represented in a reduced data set consisting of the parameters of linear fits that were calculated for twelve separate sections of the temperature cycle. These parameters, i.e., mean value and slope for each section, represent the conductance profile, forming a data set (feature vector) of $24 \mathrm{nu}-$ merical values which is used for further analysis (Schüler et al., 2015). The sections were selected manually to allow for a good representation of the temperature cycle with a small number of features, choosing longer sections for those parts of the cycle where conductance changes rather slowly and shorter sections where rapid changes occur (e.g. while the sensor is heating up from 250 to $400^{\circ} \mathrm{C}$; see Fig. S1 in the Supplement). An automated selection of these feature ranges based on objective criteria would be desirable and is the subject of our current work. We use linear discriminant analysis (LDA), a supervised algorithm for dimensionality reduction, to discriminate between different gases or poisoning states, respectively. LDA calculates a linear transformation (a set of $N-1$ so-called discriminant functions) projecting the feature vector into an $N-1$ dimensional space, with $N$ being the number of classes to be discriminated (Backhaus et al., 2000). It could be shown that ageing-induced drift has no significant influence on the discrimination of the different gases, for the test duration of $106 \mathrm{~h}$ (Schüler et al., 2015). However, HMDSO exposure does induce a strong drift in the LDA projection results. The LDA plot shown in Fig. 1 discriminates the different gas types using non-normalized data. The discriminant functions (DFs) are calculated using only data acquired before poisoning. These DFs are applied to data from all poisoning states. There is a large drift in the LDA projection after subsequent HMDSO exposures, which would obviously prevent reliable gas identification.

Figure 2 shows the result of an LDA of five classes representing the different poisoning states, based on the same data as in Fig. 1, i.e. containing all carbon monoxide and ethanol exposures at both humidity values. The poisoning is denoted by its cumulative HMDSO exposure. While a rough estimation of the poisoning state seems possible, there is a 


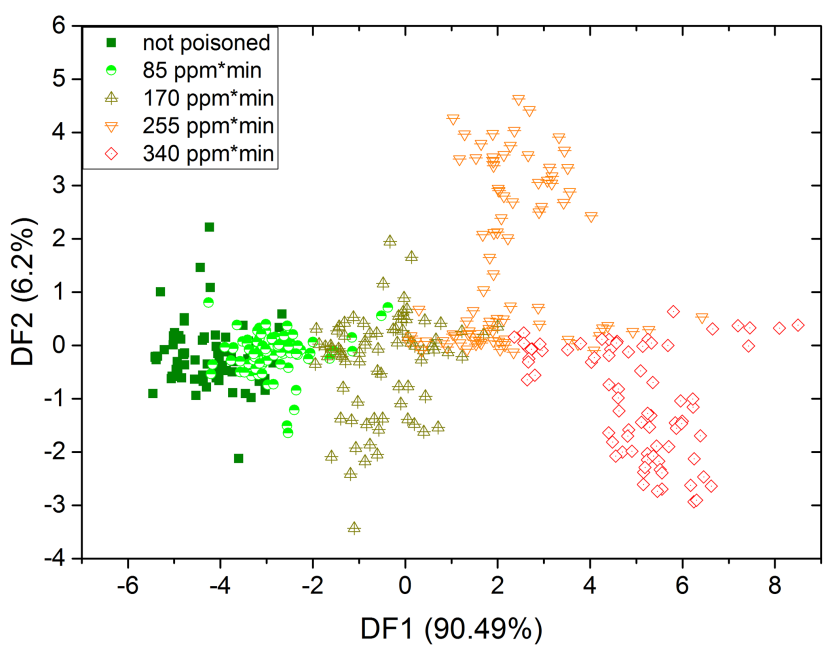

Figure 2. LDA plot showing the discrimination of different poisoning states using data from gas exposures to carbon monoxide (1/5 ppm), ethanol (1/5 ppm) and pure air, each at 40 and $60 \% \mathrm{RH}$. The 24 features used for this LDA are based on non-normalized conductance values.

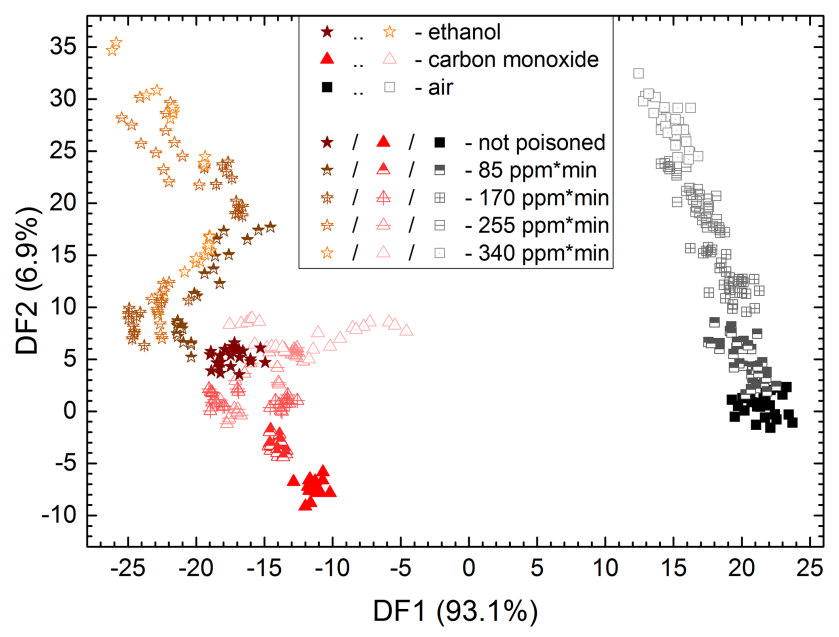

Figure 3. LDA plot showing the discrimination of different gases using data from different poisoning states. The DFs were calculated with data acquired before poisoning (solid symbols), and applied to data from all poisoning states. The 24 features used for this LDA are based on normalized conductance values, i.e. a linear projection of all conductance values of a temperature cycle to the interval $[0,1]$.

significant overlap between classes. A leave-one-out crossvalidation using the $k$-nearest neighbors $(k-\mathrm{NN})$ classifier with $k=3$ and the Euclidian distance yields a correct classification rate of $81.2 \%$; all misclassified points are attributed to a neighboring class, i.e. the poisoning state is sometimes over- or underestimated slightly (Schüler et al., 2015). The HMDSO exposures can thus be estimated with a maximum error of $\pm 85 \mathrm{ppm} \times \min$.

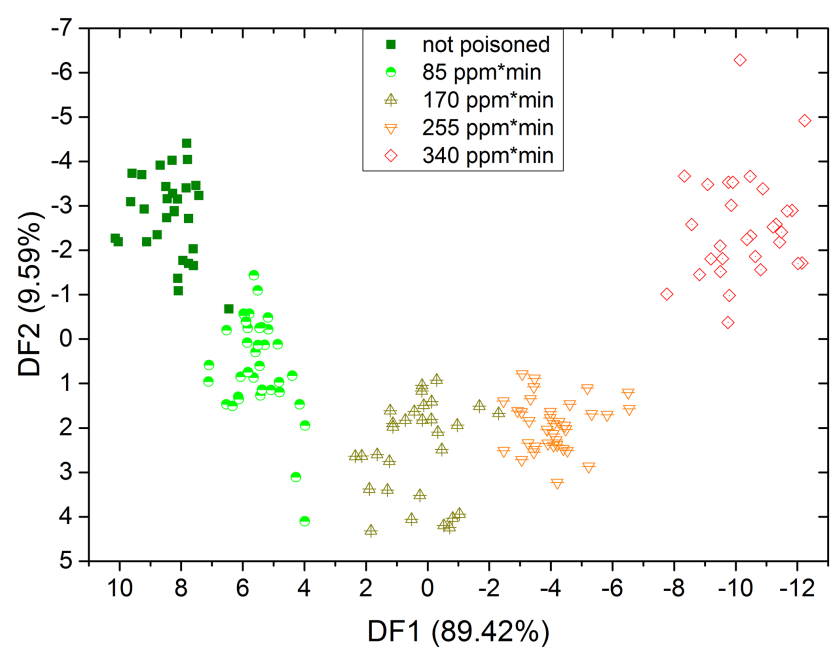

Figure 4. LDA plot showing the discrimination of different poisoning states in pure air, both at $40 \% \mathrm{RH}$ as well as $60 \% \mathrm{RH}$. The 24 features used for this LDA are based on non-normalized conductance values.

The LDA plot shown in Fig. 3 is based on normalized TCO data; otherwise the same data as shown in Fig. 1 are used. Again, the DFs are calculated using only data acquired before poisoning and are then applied to data from all poisoning states. Although drift in the LDA projection after subsequent HMDSO exposures is still evident and would prevent correct classification of the gases, the data points acquired in air are clearly separated from those in presence of reducing gases, and the overall drift is distinctly smaller compared to Fig. 1. Although the poisoning-induced sensor drift is strongly reduced by normalization, there still is an important effect of HMDSO in the shown TCO mode. It is therefore desirable to determine the degradation of the sensor accurately at early stages in order to allow for replacement of the sensor before its performance is no longer sufficient for the particular application. To this end, we examine whether the detection of poisoning in pure air is more accurate than for an arbitrary gas exposure. Thus, Fig. 4 shows an LDA plot for data acquired only in air (at both 40 and $60 \% \mathrm{RH}$ ) for classifying the five poisoning states. The classes still overlap slightly, but $97.5 \%$ are classified correctly compared to only $81.2 \%$ without prior identification of the ambient gas.

This result enables the two-step approach illustrated in Fig. 5: in a first step, we can determine if the sensor is operated in clean air or if a reducing gas is present. It has been shown that this is possible regardless of the poisoning state using normalized data (Fig. 3). In the second step, the poisoning state can be determined with high accuracy when the sensor is operated in air. This enables a precise timing of sensor replacement, and thus an optimal exploitation of the sensor lifetime. 


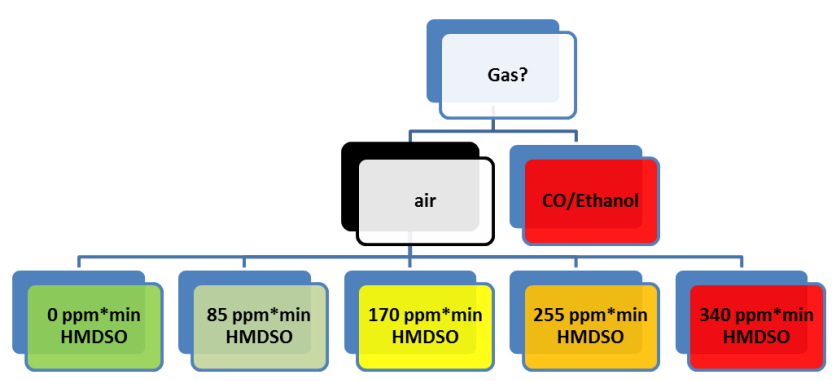

Figure 5. Hierarchical evaluation strategy for the discrimination of different poisoning states using LDA and data from pure air. A discrimination of pure air is possible using the normalized data regardless of the poisoning state (cf. Fig. 3). After determining that the sensor is operated in pure air, an accurate determination of the poisoning state is possible using non-normalized data (cf. Fig. 4). According to DIN EN 50194-1, the sensor must withstand a poisoning dose of $400 \pm 120 \mathrm{ppm} \times$ min, which implies that a detection at such early stages of poisoning is sufficient to enable a replacement before the minimum lifespan of the sensor is over.

\section{Stable sensor operation independent of HMDSO poisoning}

Based on the results achieved for the poisoning detection, we are aiming at a compensation of poisoning using TCO, i.e. a stable identification of the target gases even for strong poisoning of the sensor. To this end, another set of measurements was carried out with two new sensors of the same type (GGS 1330, UST Umweltsensortechnik GmbH). These sensors were operated using a temperature cycle developed for achieving high sensitivity (Baur et al., 2015). During the experiment, they were exposed to up to $900 \mathrm{ppm} \times \min$ HMDSO in steps of $180 \mathrm{ppm} \times$ min. After each HMDSO exposure, the reaction towards different test gases was examined, and gas discrimination was performed using LDA.

\subsection{Experimental setup}

For the following measurements, we used an automatic gas mixing system similar to the one described in Schüler et al. (2015). A schematic of the gas mixing system can be found in the supplementary material. Both test gases (carbon monoxide and ethanol) are provided from test gas cylinders. Gas flows are regulated with standard mass flow controllers. Air humidification is realized in a glass bubbler at ambient temperature $\left(25^{\circ} \mathrm{C}\right)$. HMDSO is provided from a second glass bubbler, which is temperature-regulated by a thermostat (LAUDA RE 307). An air flow of $0.5 \mathrm{~mL} \mathrm{~min}^{-1}$ is saturated with HMDSO vapor in the temperature-regulated bubbler bottle, which was kept at a constant temperature of $15^{\circ} \mathrm{C}$. It is led to a second air stream $\left(2000 \mathrm{~mL} \mathrm{~min}^{-1}\right)$ for dilution, resulting in an overall HMDSO concentration of approx. $10 \mathrm{ppm}$. During the gas measurements, gravimetric measurements of the HMDSO bottle were performed after

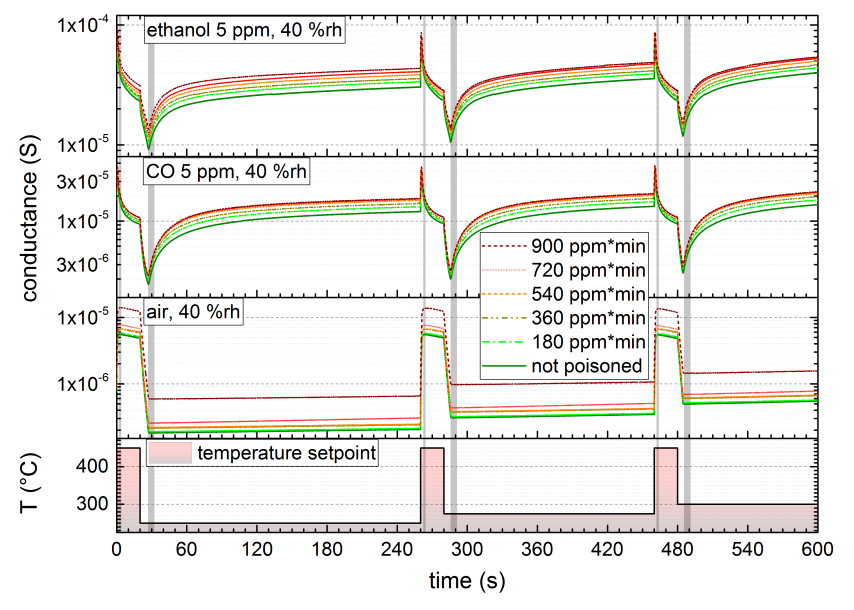

Figure 6. Temperature cycle for robust gas measurement and corresponding conductance profiles for $5 \mathrm{ppm}$ ethanol, $5 \mathrm{ppm}$ carbon monoxide and pure air, respectively, all at $40 \% \mathrm{RH}$ and for different poisoning states. The six areas shaded in gray with durations of $1 \mathrm{~s}$ for the three sections at $450{ }^{\circ} \mathrm{C}$ and $5 \mathrm{~s}$ for the three sections at lower temperatures indicate the sections of the cycle from which features (mean value and slope) were extracted for the subsequent LDA.

intervals between 24 and $25.5 \mathrm{~h}$. The resulting concentrations were consistently between 8.3 and $9.7 \mathrm{ppm}$, well within the range defined by the DIN EN 501941 standard ( $10 \pm 3 \mathrm{ppm})$.

Both sensors are exposed to the test gases as well as HMDSO. During the gas profile, which is shown in detail in the supplementary material, the sensors are exposed to 1 and $5 \mathrm{ppm}$ of carbon monoxide and ethanol at 40 and $60 \% \mathrm{RH}$, resulting in a total of eight gas exposures. Each gas exposure lasts for $60 \mathrm{~min}$, followed by $60 \mathrm{~min}$ in pure humid air. After the test gas profile the sensors are exposed to HMDSO at approx. $9 \mathrm{ppm}$ for 20 minutes in a total air flow of $2.2 \mathrm{~L} \mathrm{~min}^{-1}$ with $3.6 \% \mathrm{RH}$ corresponding to an HMDSO dose of $180 \mathrm{ppm} \times \mathrm{min}$. The gas profile is repeated 6 times after an initial $12 \mathrm{~h}$ run-in period at $40 \% \mathrm{RH}$ resulting in six poisoning levels.

\subsection{Sensor operation}

The sensors are operated with the temperature set point cycle shown at the bottom of Fig. 6. The sensor temperature is controlled using the resistance of the integrated platinum heater. Sensor temperature control and data acquisition are performed with the 3S-Toolbox (3S GmbH, Saarbrücken) sensor operation hardware for UST GGS sensors and the logarithmic amplifier described by Baur et al., 2015. The current through the sensing layer is measured every $20 \mathrm{~ms}$ at a constant voltage of $250 \mathrm{mV}$ using this logarithmic amplifier. 


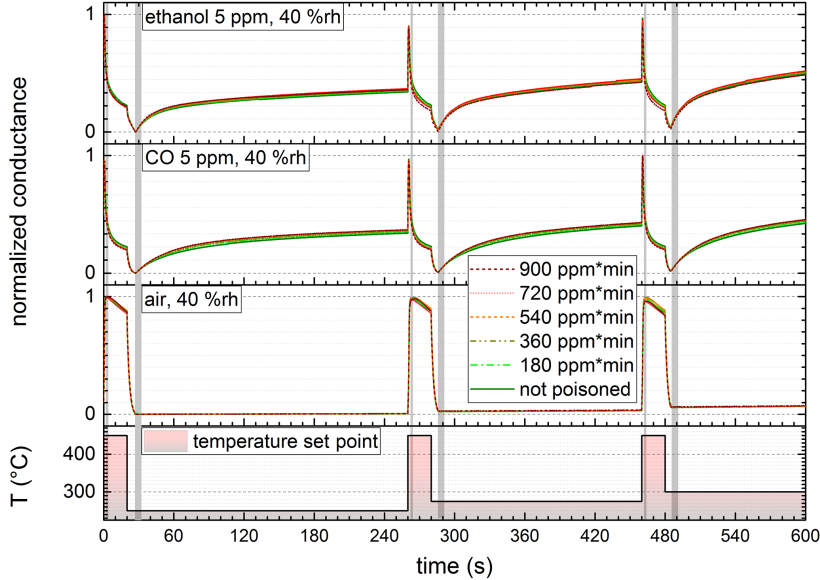

Figure 7. Temperature cycle for robust gas measurement and corresponding normalized conductance profiles for $5 \mathrm{ppm}$ ethanol, $5 \mathrm{ppm}$ carbon monoxide and pure air, respectively, all at $40 \% \mathrm{RH}$ and for different poisoning states. The six areas shaded in gray with durations of $1 \mathrm{~s}$ for the three sections at $450^{\circ} \mathrm{C}$ and $5 \mathrm{~s}$ for the three sections at lower temperatures indicate the sections of the cycle from which features (mean value and slope) were extracted for the subsequent gas discrimination LDA.

\subsection{Data analysis and results}

The conductance values over the temperature cycle are shown in Fig. 6 after different cumulative HMDSO exposures in the presence of 5 ppm ethanol, 5 ppm carbon monoxide and pure air at $40 \% \mathrm{RH}$. Again, the influence of the HMDSO exposure on the absolute conductance is obvious from the graphs, while the shape of the conductance profile remains nearly unchanged. This observation indicates that the relaxation processes observed after temperature changes as described by Baur et al., 2015, are nearly unaffected by HMDSO poisoning, as opposed to the steady state conductance. Note that the conductance values span a range of 3 orders of magnitude from $10^{-7}$ to $10^{-4} \mathrm{~S}$ even for the small gas concentrations tested in this study. This explains the requirement for the logarithmic amplifier used for data acquisition.

Figure 7 shows the influence of the normalization (linear projection of the conductance values of each temperature cycle to the interval $[0,1])$ on the conductance profile. The gray areas are used for feature extraction - the features used in the LDAs are mean values and slopes from these time intervals, representing the conductance during these intervals in a feature vector of 12 numerical values per cycle. The intervals used have a duration of $1 \mathrm{~s}$ during the hightemperature plateaus at $450^{\circ} \mathrm{C}$ and $5 \mathrm{~s}$ during the lower temperature plateaus at $250,275,300^{\circ} \mathrm{C}$, respectively. After the temperature change, a delay of $1.2 \mathrm{~s}$ at $450^{\circ} \mathrm{C}$ and $7 \mathrm{~s}$ at the lower set point temperatures was chosen to account for the thermal time constant of the sensors. These intervals were selected based on the expectation that the relaxation slopes after temperature step changes represent gas specific features

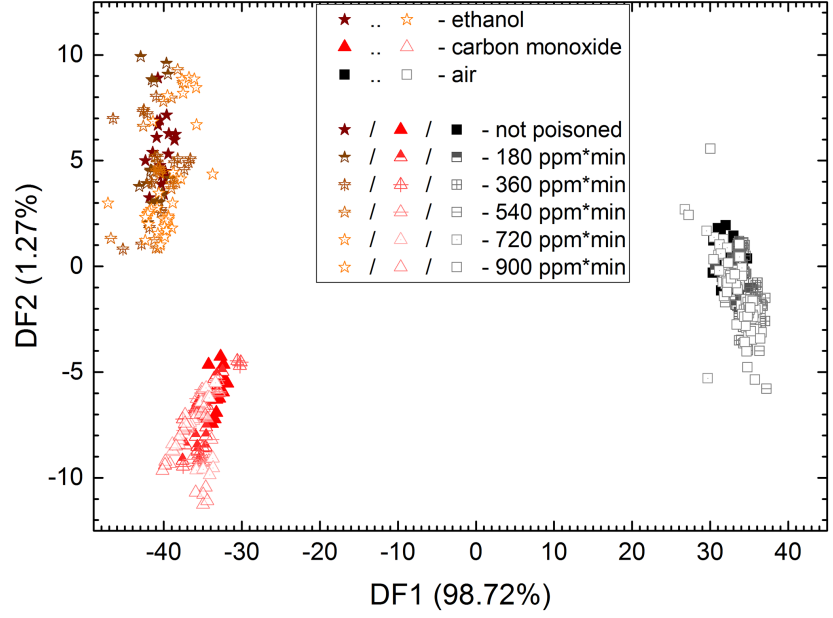

Figure 8. LDA plot showing the discrimination of different gases based on normalized data from different poisoning states of sensor 1 . The DFs were calculated with data acquired before poisoning (solid symbols), and applied to data from all poisoning states. The 12 features used for this LDA are based on normalized conductance values, i.e. a linear projection of all conductance values of a temperature cycle to the interval $[0,1]$ (cf. Fig. 7 ).

as previously reported (Baumbach et al., 2004). Furthermore, the approach presented by Baur et al., 2015, can be useful to establish shorter temperature cycles containing the same information.

For the analysis of the measurement results we again use LDA. The LDA is calculated to discriminate between the three groups pure air, carbon monoxide and ethanol. Again, concentrations of 1 and $5 \mathrm{ppm}$ are represented in one group, as well as humidities of 40 and $60 \%$ RH, i.e. the target is gas identification independent of RH and concentration. The data acquired before poisoning of the sensors are used to calculate the LDA DFs. These are applied to data from all poisoning steps resulting in the LDA plot shown in Fig. 8 for sensor 1. While the projected data groups of the unpoisoned sensor show more scatter than when using the larger feature set (compare inset of Fig. 1), the sensor drift due to poisoning is almost completely eliminated allowing $100 \%$ correct classification of all gases even after an HMDSO poisoning dose of up to $900 \mathrm{ppm} \times$ min, twice as much as demanded by DIN EN 50194-1. If at all, there is only a slight drift remaining in the LDA projection after subsequent HMDSO exposures.

For sensor 2, the corresponding result looks quite similar again, the different groups are clearly separated, and even after strong poisoning, no significant drift can be observed. The features used for these LDAs are rather specific to the gas reaction and are influenced only slightly by the poisoning. This has been verified by attempting a discrimination of different sensor poisoning states with these features: using the data from Fig. 8, i.e. normalized data from the short sections at the beginning of each temperature step, and taking into ac- 


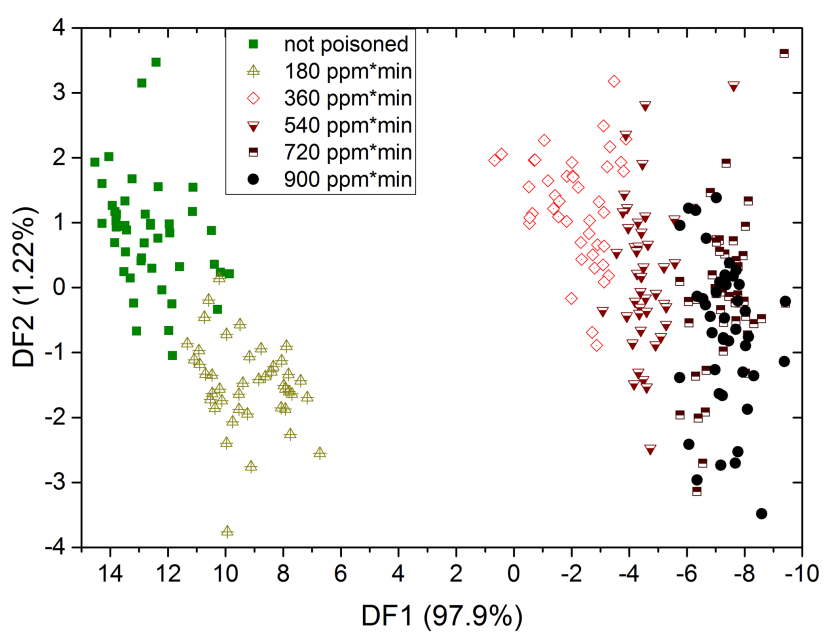

Figure 9. LDA plot showing the discrimination of different poisoning states based on data acquired during air exposure. The 12 features used for this LDA are based on linear fits of the nonnormalized conductance values during the same intervals which were also used for gas discrimination (cf. Fig. 6).

count data from all gas exposures, the resulting sensor state classification (discriminating six linearly pitched states from $0 \mathrm{ppm} \times$ min HMDSO to $900 \mathrm{ppm} \times$ min HMDSO) was correct for less than half $(48.8 \%)$ of the measurements. Using non-normalized data from the same sections, $56.6 \%$ were classified correctly. A reliable estimation of the sensor poisoning state is thus not possible, but these results confirm the improved insensitivity towards poisoning achieved through normalization. One might expect that normalization reduces the possibility to quantify the gas concentrations. However, LDAs carried out with feature sets from normalized and nonnormalized data (shown in the Supplement) disprove this assumption - the concentrations are separated even better when normalized data are used. The authors suggest that this fact is based on the influence of the gas concentration on the relaxation processes and the resulting differences in the shape of the conductance profile.

The LDA in Fig. 9 also uses non-normalized features, but only from exposure to pure air, i.e., following a two-step approach as outlined in Fig. 5. With this approach the poisoning state can be estimated, achieving an overall rate of $80.3 \%$ correctly classified sensor states. In comparison, when normalized data are used for air exposures only, the correct classification rate is $70.4 \%$. It may seem surprising that there is a relatively large gap along DF 1 between the poisoning state at $180 \mathrm{ppm} \times \min$ HMDSO and the one at $360 \mathrm{ppm} \times \min$ HMDSO. However, this is consistent with the result shown in Fig. 4, which also shows a clear separation between the poisoning state at $340 \mathrm{ppm} \times$ min HMDSO and the previous state. The evaluation of the non-normalized data used in both cases also takes the baseline conductance into account for determination of the poisoning state. Previous ex- periments on $\mathrm{SnO}_{2}$ sensors exposed to HMDSO have shown a strongly nonlinear influence of HMDSO dose on the baseline conductance. Williams and Pratt, 1998, have demonstrated that the baseline conductance increases slowly at first, and then faster with increasing HMDSO dose until it reaches a maximum before finally decreasing again. They observed a relatively sharp increase in conductance between approx. 167 and $500 \mathrm{ppm} \times \min$ HMDSO (400/1200 s exposure at $25 \mathrm{ppm}$ ), similar to our results shown in Figs. 4 and 9 where a strong shift is observed along DF1 between 255/340 and $180 / 360 \mathrm{ppm} \times \min$, respectively.

Because of the still relatively poor classification rate and the fact that the poisoning states in this classification are further apart compared to the discrimination in Fig. 4, it may be useful to switch between the temperature cycle which enables stable gas classification and one optimized for precise detection of the poisoning state, e.g. the cycle used previously. The rough estimation of the sensor state which can be provided by the temperature cycle for stable gas discrimination could then be used to determine at which time the alternative temperature cycle enabling precise detection of the sensor state should be applied.

\section{Conclusion and outlook}

Poisoning affects the conductance value of a GGS 1330 sensor significantly at two different TCO modes, i.e., using two different temperature cycles. However, the shape of the conductance during both temperature cycles is less affected by poisoning. As a consequence, normalization of the conductance profile provides signals that are significantly more stable with regard to poisoning. TCO enables monitoring of the sensor's poisoning state, allowing for quantification of the HMDSO exposure dose with an accuracy of $\pm 85 \mathrm{ppm} \times$ min (Schüler et al., 2015). Furthermore, it was shown that discrimination of pure air, carbon monoxide and ethanol independent of the gas concentration and the $\mathrm{RH}$ value is possible even after strong HMDSO exposure $(900 \mathrm{ppm} \times \mathrm{min})$, using TCO and a calibration method based only on data acquired before poisoning. This shows that in addition to its benefits for selectivity and sensitivity, TCO combined with suitable data processing can compensate poisoning with an HMDSO dose that is more than twice as high as required by the applicable industry standard (DIN EN 501941).

In particular, the longer $(600 \mathrm{~s})$ temperature cycle used here is certainly unsuitable for many practical applications due to its duration. However, similar results can be achieved using much shorter temperature cycles, which can be developed following the approach described by Baur et al. (2015). In our ongoing work, we will therefore optimize the TCO with regard to cycle duration while preserving the improvement in selectivity and stability. 


\section{The Supplement related to this article is available online at doi:10.5194/jsss-4-305-2015-supplement.}

Acknowledgements. This work is based on a project funded by the German ministry of Economic Affairs and Energy (BMWi, grant no. $16962 \mathrm{~N}$ ). The authors would especially like to thank the members of the industrial project committee for fruitful discussions and cooperation which are an important foundation of our ongoing work on sensor self-test strategies.

Edited by: F. L. Dickert

Reviewed by: two anonymous referees

\section{References}

Backhaus, K., Erichson, B., Plinke, W., and Weiber, R.: Multivariate Analysemethoden Springer-Verlag, Heidelberg, Germany, ISBN 3540-67146-3, 2000.

Baumbach, M., Kammerer, T., Sossong, A., and Schütze, A.: A new method for fast identification of gases and gas mixtures after sensor power up, Proc. IEEE Sensors, 3, 1388-1391, doi:10.1109/ICSENS.2004.1426443, 2004.

Baur, T., Schütze, A., and Sauerwald, T.: Optimierung des temperaturzyklischen Betriebs von Halbleitergassensoren/Optimization of temperature cycled operation of semiconductor gas sensors, Tech. Mess. 82, 187-195, doi:10.1515/teme-2014-0007, 2015.

DIN EN 50194-1, Electrical apparatus for the detection of combustible gases in domestic premises - Part 1: Test methods and performance requirements, German industry standard, 2009.

Ehrhardt, J.-J., Colin, L., and Jamois, D.: Poisoning of platinum surfaces by hexamethyldisiloxane (HMDS): application to catalytic methane sensors, Sensor. Actuator. B-Chem. 40, 117-124, doi:10.1016/S0925-4005(97)80250-X, 1997.

Eicker, H., Kartenberg, H. J., and Jacob, H.: Untersuchung neuer Meßverfahren mit Metalloxidhalbleitern zur Überwachung von Kohlenoxid-Konzentrationen/A study of new measuring techniques with metal oxide semiconductors designed to monitor carbon oxide concentrations, Tech. Mess. 48, 421-430, doi:10.1524/teme.1981.48.jg.421, 1981.

Gramm, A. and Schütze, A.: High performance solvent vapor identification with a two sensor array using temperature cycling and pattern classification, Sensor. Actuator. B-Chem., 95, 58-65, doi:10.1016/S0925-4005(03)00404-0, 2003.
Kohl, D.: Surface processes in the detection of reducing gases with $\mathrm{SnO}_{2}$-based devices, Sensor. Actuator. 18, 71-113, doi:10.1016/0250-6874(89)87026-X, 1989.

Kohl, D., Kelleter, J., and Petig, H.: Detection of Fires by Gas Sensors, Sensors Update 9, 161-223, doi:10.1002/16168984(200105)9:1<161::AID-SEUP161>3.0.CO;2-A, 2001.

Lalauze, R. and Pijolat, C.: A New Approach to Selective Detection of Gas by an $\mathrm{SnO}_{2}$ Solid-State Sensor, Sensor. Actuator. 5, 5563, doi:10.1016/0250-6874(84)87006-7, 1984.

Lee, A. P. and Reedy, B. J.: Temperature modulation in semiconductor gas sensing, Sensor. Actuator. B-Chem., 60, 35-42, doi:10.1016/S0925-4005(99)00241-5, 1999.

Leidinger, M., Sauerwald, T., Reimringer, W., Ventura, G., and Schütze, A.: Selective detection of hazardous VOCs for indoor air quality applications using a virtual gas sensor array, J. Sens. Sens. Syst., 3, 253-263, doi:10.5194/jsss-3-253-2014, 2014.

Meier D. C., Evju J. K., Boger, Z., Raman, B., Benkstein, K. D., Martinez, C. J., Montgomery, C. B., and Semancik, S.: The potential for and challenges of detecting chemical hazards with temperature-programmed microsensors, Sensor. Actuator. B-Chem. 121, 282-294, doi:10.1016/j.snb.2006.09.050, 2007.

Morrison, S. R.: Semiconductor gas sensors, Sensor. Actuator., 2, 329-341, doi:10.1016/0250-6874(81)80054-6, 1982.

Morrison, S. R.: Selectivity in semiconductor gas sensors, Sensor. Actuator. 12, 425-440, doi:10.1016/0250-6874(87)80061-6, 1987.

Schüler, M., Sauerwald, T., and Schütze, A.: Metal oxide semiconductor gas sensor self-test using Fourier-based impedance spectroscopy, J. Sens. Sens. Syst., 3, 213-221, doi:10.5194/jsss-3213-2014, 2014.

Schüler, M., Fricke, T., Sauerwald, T., and Schütze, A.: Detecting poisoning of metal oxide gas sensors at an early stage by temperature cycled operation, AMA Conferences 2015 - Proceedings, Nuremberg, Germany, 19-21 May 2015, 735-740, doi:10.5162/sensor2015/E8.4, 2015.

Schütze, A., Gramm, A., and Rühl, T., Identification of Organic Solvents by a Virtual Multisensor System with Hierarchical Classification, IEEE Sens. J., 4, 857-863, doi:10.1109/JSEN.2004.833514, 2004.

Williams, D. E.: Semiconducting oxides as gas-sensitive resistors, Sensor. Actuator. B-Chem., 57, 1-16, doi:10.1016/S09254005(99)00133-1, 1999.

Williams, D. E. and Pratt, F. E.: Classification of reactive sites on the surface of polycrystalline tin dioxide, J. Chem. Soc., 94, 34933500, doi:10.1039/A807644H,1998. 